\title{
On the role of a new type of correlated disorder in extended electronic states in the Thue-Morse lattice
}

\author{
Arunava Chakrabarti円, S. N. Karmakar and R. K. Moitra \\ Saha Institute of Nuclear Physics, 1/AF, Bidhannagar, Calcutta 700 064, \\ India.
}

\begin{abstract}
A new type of correlated disorder is shown to be responsible for the appearance of extended electronic states in one-dimensional aperiodic systems like the Thue-Morse lattice. Our analysis leads to an understanding of the underlying reason for the extended states in this system, for which only numerical evidence is available in the literature so far. The present work also sheds light on the restrictive conditions under which the extended states are supported by this lattice.
\end{abstract}

PACS Nos.: 61.44.+p, 64.60.Ak, 71.20.Ad, 71.25.-s

\footnotetext{
${ }^{1}$ Permanent Address : Scottish Church College, 1 \& 3 Urquhart Square, Calcutta 700 006, India.
} 
The role of correlated disorder in producing extended electronic states in one-dimensional disordered systems has been extensively discussed in the literature by now [1]-[3]. The basic reason for the appearance of extended states in such cases has been traced to the existence of a certain type of short range clustering effect among the atoms, first pointed out by Dunlap et al. [1] in their study of a distribution of random dimers on a host lattice. It was shown that at a certain energy value the composite transfer matrix for a dimer offers identity contribution to the full transfer matrix, so that at this energy the full disordered lattice effectively behaves as an ordered chain. These correlations have later been shown to persist at all length scales in several quasiperiodic lattices like the copper mean chain and the period doubling lattice [2], leading to whole hierarchies of extended states.

Instances of one-dimensional lattices are however known which offer more intriguing possibilities. One such example is the well-known Thue-Morse aperiodic sequence, in which there is numerical evidence of extended states [4] although there is no short range dimer-type positional correlations in this lattice. As we shall see in this paper, another kind of clustering effect manifests itself in this lattice, which cannot be analysed by the standard method known so far and developed in detail in Ref. [1] and [2]. We may mention in this connection the recent work of Lin and Goda [5] concerning a problem of hierarchical distribution of potentials on a lattice, where they also find an infinite number of extended electronic eigenstates, although there are no dimer-type correlations. The present work therefore provides yet another example of an aperiodic lattice where the standard picture of correlated disorder does not work.

In a recent work on the Thue-Morse lattice, Ryu et al. [4] have provided numerical evidence of the existence of extended electronic eigenstates at certain specified energies, based on the study of the trace map for this lattice. It has been shown that a particular value of the trace remains unchanged under a renormalisation group transformation. By using a detailed multifractal analysis they found that the energies associated with this trace value correspond to extended eigenstates. This work, however, does not provide any analytical guideline for discerning the extended character of these eigenfunctions, and the physical mechanism underlying the behaviour of these eigenfunctions, independently of any multifractal analysis, remains to be clarified. The main purpose of this work is to provide this guideline. Additionally, we demonstrate the restrictive conditions under which this lattice supports 
extended states. We show that extended states are supported on this lattice only within the framework of the on-site model; the other commonly used model, namely the bond-model, does not support extended states, so that our work invalidates the findings of Ref. [6] regarding the analysis of a multiband model of the Thue-Morse lattice. A rigorous repudiation of the results of Ryu et al. has recently also been given by Bovier and Ghez [7].

A Thue-Morse (TM) chain may be built up by starting with two letters $A$ and $B$ and using the inflation rule $A \rightarrow A B$ and $B \rightarrow B A$ repeatedly, where the letters $A$ and $B$ may be thought of as representing the atoms $A$ and $B$. The ratio of the numbers of $A$ and $B$ atoms in any generation $\ell$ is $N_{A}(\ell) / N_{B}(\ell)=1$. The electronic properties of such a lattice are generally modelled by the on-site version of the nearest-neighbour hamiltonian in the Wannier basis, the Schrodinger equation corresponding to which is given by

$$
\left(E-\epsilon_{n}\right) \psi_{n}=\psi_{n+1}+\psi_{n-1}
$$

$n$ being the site index, and $\epsilon_{n}=\epsilon_{A}$ or $\epsilon_{B}$, and the energies are in units of the hopping integral $t$ which has been set equal to unity.

The amplitudes of the wave functions at different atomic sites are determined by using the unimodular transfer matrices of the form

$$
M_{n}=\left(\begin{array}{cc}
E-\epsilon_{n} & -1 \\
1 & 0
\end{array}\right) .
$$

The amplitude of the wave function at the $n$-th site is then related to that at the first site by the following matrix product

$$
M_{n} M_{n-1} \cdots \cdots M_{1}
$$

In order to unravel the correlations that are responsible for the extended states in a Thue-Morse chain, we first recapitulate the structural peculiarities of this lattice. At the very basic level, we may regard the atoms $A$ and $B$ to be the basic building blocks of the TM lattice; at the next level the pairs $A B$ and $B A$ may be regarded as the basic starting elements on which the repeated application of the Thue-Morse inflation rules generates the whole lattice. We may successively consider the pair of quadruplets $A B B A$ and $B A A B$, the pair $A B B A B A A B$ and $B A A B A B B A$, the pair $A B B A B A A B B A A B A B B A$ and $B A A B A B B A A B B A B A A B$ etc. as the starting blocks for generating 
the Thue-Morse chain by applying the appropriate inflation rules. Evidently, there are no dimer or higher order atomic clustering in this lattice. It thus becomes necessary to analyse the products of transfer matrices corresponding to these strings of atoms in order to understand the conditions under which extended states are supported by the TM lattice.

To handle such long products of matrices we resort to the following device. Let us begin by resolving the $2 \times 2$ matrices $M_{n}$ in a basis formed by the $2 \times 2$ identity matrix $I$ and the three Pauli matrices $\sigma_{x}, \sigma_{y}$ and $\sigma_{z}$. We then have the expressions for $M_{A}$ and $M_{B}$ in the following form:

$$
\begin{aligned}
& M_{A}=\alpha_{A} I+\beta_{A} \sigma_{x}+\gamma_{A} \sigma_{y}+\delta_{A} \sigma_{z} \\
& M_{B}=\alpha_{B} I+\beta_{B} \sigma_{x}+\gamma_{B} \sigma_{y}+\delta_{B} \sigma_{z}
\end{aligned}
$$

where $\alpha_{A(B)}=\delta_{A(B)}=\left(E-\epsilon_{A(B)}\right) / 2, \beta_{A(B)}=0$ and $\gamma_{A(B)}=-i$.

Using Eqs.(3) and the commutation rules for the matrices $\sigma_{x}, \sigma_{y}$ and $\sigma_{z}$, we may easily resolve the matrix products $M_{A} M_{B}$ and $M_{B} M_{A}$ to obtain

$$
\begin{aligned}
& M_{A} M_{B}=\alpha_{1} I+\beta_{1} \sigma_{x}+\gamma_{1} \sigma_{y}+\delta_{1} \sigma_{z} \\
& M_{B} M_{A}=\alpha_{1} I-\beta_{1} \sigma_{x}+\gamma_{1} \sigma_{y}+\delta_{1} \sigma_{z}
\end{aligned}
$$

where $\alpha_{1}=2 \alpha_{A} \alpha_{B}-1, \beta_{1}=\alpha_{B}-\alpha_{A}, \gamma_{1}=-i\left(\alpha_{A}+\alpha_{B}\right)$ and $\delta_{1}=2 \alpha_{A} \alpha_{B}$. We notice that the coefficients of the matrices $I, \sigma_{y}$ and $\sigma_{z}$ are the same for both the products $M_{A} M_{B}$ and $M_{B} M_{A}$ in Eqs.(田), while the coefficient of $\sigma_{x}$ in these expressions appear with opposite signs. Thus if we set this coefficient, ie. $\beta_{1}=0$, then the products $M_{A} M_{B}$ and $M_{B} M_{A}$ become identical, and the system effectively reduces to an ordered binary chain of alternating $A$ and $B$ atoms. However, it turns out that the condition $\beta_{1}=0$ can only be satisfied with $\epsilon_{A}=\epsilon_{B}$. We thus have to proceed to the next stages, and compare the pairs of matrix products $M_{A} M_{B} M_{B} M_{A}$ and $M_{B} M_{A} M_{A} M_{B}$, the products $M_{A} M_{B} M_{B} M_{A} M_{B} M_{A} M_{A} M_{B}$ and $M_{B} M_{A} M_{A} M_{B} M_{A} M_{B} M_{B} M_{A}$ and so on.

Using the basic results for the resolutions of the matrices $M_{A}$ and $M_{B}$ (Eqs.(3)) and for the products $M_{A} M_{B}$ and $M_{B} M_{A}$ (Eqs.(四)) repeatedly, we can easily find the forms of the longer matrix products mentioned above. Interestingly, the pair of products of matrices $M_{A} M_{B} M_{B} M_{A} M_{B} M_{A} M_{A} M_{B}$ 
$\ldots$ and $M_{B} M_{A} M_{A} M_{B} M_{A} M_{B} M_{B} M_{A} \ldots$ each with $2^{n}$ elements show a surprising regularity having either of the following forms

$$
\begin{aligned}
& M_{A} M_{B} M_{B} M_{A} M_{B} M_{A} M_{A} M_{B} \cdots=\alpha_{n} I+\gamma_{n} \sigma_{y}+\delta_{n} \sigma_{z} \\
& M_{B} M_{A} M_{A} M_{B} M_{A} M_{B} M_{B} M_{A} \cdots=\alpha_{n} I+\gamma_{n}^{\prime} \sigma_{y}+\delta_{n}^{\prime} \sigma_{z}
\end{aligned}
$$

and

$$
\begin{aligned}
& M_{A} M_{B} M_{B} M_{A} M_{B} M_{A} M_{A} M_{B} \cdots=\alpha_{n} I+\beta_{n} \sigma_{x}+\gamma_{n} \sigma_{y}+\delta_{n} \sigma_{z} \\
& M_{B} M_{A} M_{A} M_{B} M_{A} M_{B} M_{B} M_{A} \cdots=\alpha_{n} I-\beta_{n} \sigma_{x}+\gamma_{n} \sigma_{y}+\delta_{n} \sigma_{z}
\end{aligned}
$$

It follows that the matrix products $M_{A} M_{B} M_{B} M_{A} M_{B} M_{A} M_{A} M_{B} \cdots$ and $M_{B} M_{A} M_{A} M_{B} M_{A} M_{B} M_{B} M_{A} \cdots$ can never be made equal at some value of the energy for even values of $n$ because their expansions given in Eqs.(5) differ in two coefficients $\gamma$ and $\delta$. On the other hand, for odd values of $n$, Eqs.(6) show that the matrix products become equal to each other if $\beta_{n}=0$. Thus the energy values for which $\beta_{n}=0$ ( $n$ odd) are the ones for which the composite transfer matrices for of the strings of atoms $A B B A B A A B \ldots$ and $B A A B A B B A \cdots$ offer identical contributions to the full transfer matrix for the whole chain. Since the Thue-Morse chain may be regarded as built out of these composite blocks, the full transfer matrix for the whole chain now consists of a product of identical unimodular $2 \times 2$ matrices $M$, each corresponding to a block of atoms $A B B A B A A B \cdots$ or $B A A B A B B A \cdots$. This however does not mean that one necessarily has extended states at all the energies obtained by setting $\beta_{n}=0$. Several of these energies in fact lead to diverging, and therefore disallowed functions, and a careful analysis has to be made to determine precise conditions under which the string of such identical matrices leads to extended states, as the length of the chain becomes arbitrarily large.

We first give the recursion relations connecting the coefficients $\alpha, \beta, \gamma$ and $\delta$ for two successive odd values of $n(n \geq 3)$ which are easily obtained 
by using Eqs.(5) and Eqs.(6). These are

$$
\begin{aligned}
\alpha_{n}= & \alpha_{n-2}^{4}+\beta_{n-2}^{4}+\gamma_{n-2}^{4}+\delta_{n-2}^{4}+2\left(\gamma_{n-2}^{2}+\delta_{n-2}^{2}\right)\left(3 \alpha_{n-2}^{2}+\beta_{n-2}^{2}\right) \\
& +2\left(\gamma_{n-2}^{2} \delta_{n-2}^{2}-\alpha_{n-2}^{2} \beta_{n-2}^{2}\right) \\
\beta_{n}= & 8 \alpha_{n-2} \beta_{n-2}\left(\gamma_{n-2}^{2}+\delta_{n-2}^{2}\right) \\
\gamma_{n}= & 4\left(\alpha_{n-2}^{2}-\beta_{n-2}^{2}+\gamma_{n-2}^{2}+\delta_{n-2}^{2}\right) \alpha_{n-2} \gamma_{n-2} \\
\delta_{n}= & 4\left(\alpha_{n-2}^{2}-\beta_{n-2}^{2}+\gamma_{n-2}^{2}+\delta_{n-2}^{2}\right) \alpha_{n-2} \delta_{n-2}
\end{aligned}
$$

From Eqs.(1) we find that the condition $\beta_{n}=0$ implies either of the following conditions:

$$
\begin{aligned}
\alpha_{n-2} & =0 \\
\beta_{n-2} & =0 \\
\gamma_{n-2}^{2}+\delta_{n-2}^{2} & =0 .
\end{aligned}
$$

First, it can be easily shown with the help of the recursion relations (7) that the value of $\alpha_{n}=1$ in each of these cases. Among the three conditions Eqs.(8-10), only the condition $\alpha_{n-2}=0$ automatically leads to $\gamma_{n}=0$ and $\delta_{n}=0$, while the two other conditions (9) and (10) generally yield non-zero values of $\gamma_{n}$ and $\delta_{n}$. As will be shown presently, the first condition always leads to allowed values of the energies for the extended states in the TM lattice. The energies obtained from the two other conditions, Eqs.(9) and (10), generally lead to asympotically diverging functions, except in special circumstances to be discussed later.

To carry out this analysis, let us express the matrix $M$ at any level in the form ( suppressing the index $n$ )

$$
M=\left(\begin{array}{cc}
\alpha+\delta & \beta-i \gamma \\
\beta+i \gamma & \alpha-\delta
\end{array}\right)
$$

$\alpha$ can be identified with the quantity $(1 / 2) \operatorname{Tr} M$. We now recall the well-known result [8] for the powers of $2 \times 2$ unimodular matrices $M$

$$
M^{m}=U_{m-1}(x) M-U_{m-2}(x) I
$$


for $m \geq 2$. Here $U_{m}(x)$ is the Chebyshev polynomial of the second kind, and $x$ is the quantity $(1 / 2) \operatorname{Tr} M=\alpha$. The function $U_{m}(x)$ is defined as $\sin (m+1) \theta / \sin \theta$ with $\cos \theta=x$. In the present discussion $\alpha_{n}=1$ in every case, for which $U_{m}(1)$ has the limiting value $(m+1)$. We then have the following expression for $M^{m}(x=1)$ :

$$
M^{m}=\left(\begin{array}{cc}
m(\alpha+\delta)-m+1 & m(\beta-i \gamma) \\
m(\beta+i \gamma) & m(\alpha-\delta)-m+1
\end{array}\right) .
$$

Let us now choose, without any loss of generality, the amplitudes of the wavefunctions at the zero-th and the first site to be 0 and 1 respectively. With the aid of Eq.(11) it then becomes simple to express the amplitudes at the $(m+1)$-th and the $m$-th sites as,

$$
\psi_{m+1}=m(\alpha+\delta-1)+1 \quad \text { and } \quad \psi_{m}=m(\beta+i \gamma) .
$$

We now observe that the finiteness of $\psi_{m}$ at $m \rightarrow \infty$ is assured only when $\alpha+\delta=1$ and $\beta+i \gamma=0$ simultaneously. Now, as we have already seen, having $\alpha_{n-2}=0$ at some stage automatically makes both $\gamma_{n}$ and $\delta_{n}$ equal to zero, in addition to making $\alpha_{n}=1$. Therefore, the criterion for the finiteness of the wavefunction at $m \rightarrow \infty$ is satisfied in this case. On the other hand, since neither of the conditions Eqs.(9) and (10) yield $\gamma_{n}$ and $\delta_{n}$ equal to zero, so that even though $\alpha_{n}=1$, the amplitudes diverge with increasing $m$. The energies obtained from these conditions may either not lead to proper eigenstates for the system or they may correspond to eigenstates with power law growth at infinity. An exception occurs when in condition (10) the quantities $\gamma_{n-2}$ and $\delta_{n-2}$ possess common factors, so that energy values exist for which $\gamma_{n-2}$ and $\delta_{n-2}$ become zero simultaneously. In this case $\gamma_{n}$ and $\delta_{n}$ become zero and we again have extended states at these energies.

Hence we arrive at the condition $\alpha_{n-2}=0$ that gives rise to a set of energy eigenvalues which unambiguously correspond to extended eigenstates for the infinite TM lattice. Each block $M_{A} M_{B} M_{B} M_{A} M_{B} M_{A} M_{A} M_{B} \cdots$ or $M_{B} M_{A} M_{A} M_{B} M_{A} M_{B} M_{B} M_{A} \cdots$ of size $2^{n}$ ( $n$ odd ) at these energy eigenvalues becomes an identity matrix and therefore the total transmission across the chain becomes unity at these energies. For all other energy values arising out of the conditions (9) and (10), which of course also correspond to the basic condition $\beta_{n}=0, \psi_{m}$ grows with $m$, and therefore they are not permissible. As mentioned above, the only exception occurs when $\gamma_{n-2}$ and $\delta_{n-2}$ 
have common factors and therefore vanish for the same value of the energy at the same time.

Although it is straighforward to obtain the algebraic expressions for the energy values obtained from the condition $\alpha_{n-2}=0$, we calculate instead the energies for a specific choice of the parameter values, namely $\epsilon_{A}=-\epsilon_{B}=0.5$. We then obtain the following energy values for the first two levels :

$$
\begin{aligned}
n=3: & \pm 1.5 \\
n=5: & \pm 2.094225204000, \pm 1.706503280648 \\
& \pm 1.260097834749, \pm 0.337965671230
\end{aligned}
$$

For the sake of illustration we display in Fig.1 the variation of $|\psi|^{2}$ with the site-index $n$ for a few selected energies. The signature of the underlying Thue-Morse aperiodicity is particularly apparent in Figs.1(a), 1(c) and 1(d); all the wave-functions are lattice-like. The general characteristic of these functions is that with increasing $n$, amplitudes tend to cluster around groups of sites separated by islands where the amplitudes have very low values, a fact which was also pointed out by Ryu et al. [6].

The spirit in which the present analysis has been carried out corresponds to the real space renormalisation group point of view. At any length scale corresponding to an odd value of $n$, Eqs.(7) define effective $A$ and $B$ atoms arranged in a TM sequence. The extended character of the eigenfunctions discussed in this paper owe their origin to a peculiar short range correlation among the atoms at every length scale, in contrast to the usual dimer-type short range correlation discussed so far in the literature.

The analysis so far has been made on the basis of the on-site model of the TM lattice. In dealing with the pure transfer model in which we have two different hopping strengths for the long $(L)$ and short $(S)$ bonds, it turns out that the full transfer matrix for the chain is composed of a product of four different transfer matrices corresponding to the $L L, L S, S L$ and $S S$ pairs. This is in contrast to the unique pair of $2 \times 2$ matrices that can act as "building blocks" for the entire TM chain in the on-site case. The four basic transfer matrices do not follow the TM sequence and form an intractable complex pattern, so that the recursive application of the TM inflation rule is not meaningful in this case. It therefore follows that the analysis of strings of transfer matrices based on Eqs.(5) and (6) is no longer possible, and thus there are no extended states in the transfer model. We may point out that 
the matrix recursion relation employed by Ryu et al. [6], $M_{l}=M_{l-1} \bar{M}_{l-1}$ is incorrect for the transfer model, because the string of matrices do not take into account the atomic site flanked by a pair of $S-S$ bond and a pair of $L-L$ bond on both sides. Here, $M_{l}$ is the transfer matrix for the $l$-th generation chain, and $\bar{M}_{l}$ is the same matrix $M_{l}$ with the roles of $t_{L}$ and $t_{S}$ interchanged. It can be verified easily that the above mapping does not generate the correct TM chain in the transfer model. Thus the analysis of Ref. [6] of the multiband model based on the above mapping is not valid. In fact, for mixed models involving variations in both site-energies and the hopping, we still have four different transfer matrices, and again there are no extended states.

To conclude, we have demonstrated a new mechanism through which short range correlations lead to extended states in aperiodic one dimensional chains such as the TM lattice. For this, we provide a way of analysis which hopefully will be found useful in problems of similar kind. 


\section{References}

[1] D. H. Dunlap, H. L. Wu and P. Phillips, Phys. Rev. Lett. 65, 88 (1990); H. L. Wu and P. Phillips, Phys. Rev. Lett. 66, 1366 (1991)

[2] S. Sil, S. N. Karmakar, R. K. Moitra and A. Chakrabarti, Phys. Rev. B 48, 4192 (1993); A. Chakrabarti, S. N. Karmakar and R. K. Moitra, Phys. Rev. B 50, 13276 (1994)

[3] A. Sanchez, E. Macia and E. Dominguez-Adame, Phys. Rev. B 49, 147 (1994)

[4] C. S. Ryu, G. Y. Oh and M. H. Lee, Phys. Rev. B 46, 5162 (1992)

[5] Z. Lin and M. Goda, J. Phys. A: Math. Gen. 26, L217 (1993)

[6] C. S. Ryu, G. Y. Oh and M. H. Lee, Phys. Rev. B 48, 132 (1993)

[7] A. Bovier and J. M. Ghez (private communication)

[8] A. Cayley, Philos. Trans. Roy. Soc. (London) 148, 17 (1858); F. Abeles, Ann. de Physique 5, 777 (1950); for a recent presntation see J. M. Luck, "Aperiodic structures: geometry, diffraction spectra and physical properties" in Fundamental Problems in Statistical Mechanics VIII, Elsevier (1994) 


\section{Figure Caption}

Fig.1 Plot of $\left|\psi_{n}\right|^{2}$ versus site number $n$ for a TM sequence with 256 sites. Here $\epsilon_{A}=-\epsilon_{B}=0.5$ with energies measured in units of hopping matrix element $t$. Figs.(a), (b), (c) and (d) correspond respectively to energies 1.5, $0.337965671230,-1.260097834749$ and -1.995507972823 . 